\title{
Cancer-Associated Fibroblasts and Desmoplastic Reactions Related to Cancer Invasiveness in Patients With Colorectal
} Cancer

\author{
Nari Shin ${ }^{1}$, Gyung Mo Son ${ }^{2,3}$, Dong-Hoon Shin ${ }^{4}$, Myeong-Sook Kwon ${ }^{2,3}$, Byung-Soo Park ${ }^{2}$, Hyun-Sung Kim², \\ Dongryeol Ryu ${ }^{5}$, Chi-Dug Kang ${ }^{6}$ \\ ${ }^{1}$ Department of Pathology, Hanyang University Hanmaeum Changwon Hospital, Changwon; ${ }^{2}$ Department of Surgery, Pusan National University \\ Yangsan Hospital, Yangsan; ${ }^{3}$ Research Institute for Convergence of Biomedical Science and Technology, Pusan National University Yangsan, \\ Yangsan; ${ }^{4}$ Department of Pathology, Pusan National University Yangsan Hospital, Yangsan; ${ }^{5}$ Department of Korean Medical Science, School of \\ Korean Medicine, Pusan National University, Busan; ${ }^{6}$ Department of Biochemistry, Pusan National University School of Medicine, Busan, Korea
}

Purpose: We evaluated the relationship of cancer-associated fibroblasts (CAFs) and desmoplastic reactions with cancer invasiveness and long-term outcomes in patients with colorectal cancer (CRC).

Methods: Histologic evaluation of mature CAFs and desmoplasia was performed by observing the collagen fiber structure and fibroblast cytomorphology in the intratumoral stroma and invasive front of CRC tissues. Cancer-cell invasiveness was evaluated using lymphatic invasion, vascular invasion, perineural invasion, tumor budding, and tumor growth patterns. Overall survival and systemic recurrence were analyzed. A network analysis was performed between CAF maturation, desmoplastic reaction, and cancer invasiveness.

Results: The proportions of mature CAFs in the intratumoral stroma and the invasive front were $57.6 \%$ and $60.3 \%$, respectively. Epidermal growth factor receptor (EGFR) overexpression was significantly higher in the mature CAFs in the invasive front as compared to immature CAFs. Lymphatic invasion increased as the number of mature fibroblasts in the intratumoral stroma increased. Tumor budding was observed in almost half of both mature and immature stroma samples and occurred more frequently in infiltrating tumors. On network analysis, well-connected islands were identified that was associated with EGFR overexpression, CAF maturation, and infiltrating tumor growth patterns leading to tumor budding. Conclusion: The maturity of CAFs and desmoplastic reactions were associated with cancer invasion. However, the cytomorphologic characteristics of CAFs were insufficient as an independent prognostic factor for patients with CRC.

Keywords: Cancer-associated fibroblasts; Desmoplasia; Survival; Wound healing; Colorectal neoplasms

\section{INTRODUCTION}

Colon cancer is one of the most common gastrointestinal cancers

Received: July 23, 2018 - Accepted: September 10, 2018

Correspondence to: Gyung Mo Son, M.D.

Department of Surgery and Research institute for Convergence of Biomedical Science and Technology, Pusan National University Yangsan Hospital, 20 Geumo-ro, Mulgeum-eup, Yangsan 50612, Korea

Tel: +82-55-360-2124, Fax: +82-55-360-2154

E-mail:skm1711@pusan.ac.kr

ORCID code: https://orcid.org/0000-0002-8861-6293

(c) 2019 The Korean Society of Coloproctology

This is an open-access article distributed under the terms of the Creative Commons Attribution NonCommercial License (http://creativecommons.org/licenses/by-nc/4.0) which permits unrestricted noncommercial use, distribution, and reproduction in any medium, provided the original work is properly cited. worldwide. Early diagnosis and treatment of patients with colorectal cancer have improved the survival rate, but $60 \%$ of patients with colorectal cancer still have metastasis to the liver or lung [1]. Metastasis is a multistep process that requires changes in tumor cell biology. However, recent findings on the tumor microenvironment have shifted the paradigm, and we now know that the host response plays a significant role in this process. During cancer invasion and metastasis, cancer cells are affected by various cells in the tumor microenvironment [2]. Moreover, a variety of histologic reactions can occur around the tumor.

Fibrosis is a normal reaction that occurs during wound healing, and regions of mature fibrosis with dense collagen fibers are expected to act as barriers to inhibit the spread of cancer cells. The desmoplastic reactions around the tumor can show a histological 
structure similar to wound healing and has been reported as a favorable prognostic factor in patients with colorectal cancer $[3,4]$. However, immature desmoplastic reactions are histologically similar to keloid scars and can promote tumor infiltration, indicating an undesirable prognosis $[5,6]$. Cancer-associated fibroblast (CAF) activity, regulated by various growth factors from cancer cells, was recently found to promote cancer cell metabolism, proliferation, and metastasis [7-10]. Activated fibroblasts produce abundant collagen fibers to form high-density fibrosis around cancer cells, and fiber contraction with increasing tumor stiffness can promote cancer cell invasion [11-14]. Experimental studies of CAF activation and cancer invasion have raised questions that are still not fully understood. Thus, to date, only few studies have directly assessed the effects of CAFs and the desmoplastic responses on cancer invasion in pathologic tissues from patients with colon cancer. Thus, the purpose of this study was to evaluate the relationships of CAFs and desmoplastic reactions to cancer invasiveness and long-term oncologic outcomes in patients with colorectal cancer.

\section{METHODS}

\section{Patients and methods}

We randomly selected 151 patients from a prospectively recorded cohort of colorectal cancer patients who underwent a follow-up of 3 years or longer. This study was conducted after receiving the approval of the Institutional Review Board (approval number: 052016-097) of the Pusan National University Yangsan Hospital. Written informed consent was obtained from all patients included in this study.

Clinicopathologic characteristics and survival data were collected through outpatient records and telephone visits. Clinical factors included age, gender, body mass index, serum carcinoembryonic antigen (CEA), and colon obstruction. Pathologic factors included tumor size, pathologic TNM stage, and cancer cell differentiation. Tumor size was measured in the 2 dimensions of the formalin-fixed tumor. TNM staging was classified according to the criteria of the 7th edition of the American Joint Committee on Cancer [15]. The 3-tier system of well (G1), moderate (G2) and poor (G3) differentiation was applied according to World Health Organization histologic grade.

Molecular biological factors, such as cytoplasmic CEA accumulation, p53 accumulation in the cancer cell, epidermal growth factor receptor (EGFR) overexpression, K-ras proto-oncogene (KRAS) mutation, B-raf proto-oncogene (BRAF) mutation, and microsatellite instability (MSI), were assessed. EGFR (mouse monoclonal antibody, clone: E30, M7239, DAKO, Glostrup, Denmark), CEA (mouse monoclonal antibody, clone: II-7, DAKO), and p53 (mouse monoclonal antibody, clone: DO-7, NCL-L-p53-DO7, Novocastra, Newcastle upon Tyne, UK) were identified by immunohistochemical staining. EGFR expression was considered positive when it was recognized in more than $10 \%$ of the cancer cells.
Cytoplasmic CEA accumulation was considered positive in cases where more than $10 \%$ of the tumor cells showed cytoplasmic staining. Nuclear accumulation of p53 was considered positive when it was recognized in more than $10 \%$ of the tumor cells. KRAS (exon 12 and 13) and BRAF (exon v600E) mutations were detected by using the quantitative real-time reverse transcription polymerase chain reaction with paraffin block tissue [16]. MSIhigh was defined as instability in at least 2 of the 5 microsatellite loci, MSI-low as instability in only one locus, and microsatellite stable as instability in no loci.

\section{Maturation of desmoplasia and fibroblasts}

Hematoxylin \& eosin (H\&E) staining was used to assess the maturation of desmoplasia and fibroblasts. H\&E staining was also used to evaluate the morphology of stromal cells and the extracellular matrix to classify the histologic changes in the tumor stroma. Stromal response to cancer cells was classified according to the dominant characteristics of stromal tissue. Tissue in which a collagen structure and fibroblasts were predominantly distributed in the tumor matrix was classified as a desmoplastic reaction, and tissue in which inflammatory cells predominated as an inflammatory reaction. If mucous accounted for more than $50 \%$ of the stroma, this was defined as a mucinous reaction. Histologic evaluations for desmoplasia and fibroblast maturation were performed by observing the collagen fiber's structure and fibroblast cytomorphology. Mature stroma was composed of elongated collagen fibers and spindle-shaped fibroblasts, and immature stroma was composed of keloid-like collagen bundles and plump fibroblasts. CAF maturity was categorized according to the cytomorphological characteristics. Thin, wavy spindle cells were classified as mature CAFs, and large fibroblasts with plump nuclei were classified as immature CAFs (Fig. 1). The maturity of the desmoplastic reaction was measured at the intratumoral stroma and the invasive front and was classified according to the report of Ueno et al. [3].

\section{Evaluation of cancer invasiveness}

To evaluate the invasiveness of cancer cells, we examined the $\mathrm{T}$ status, $\mathrm{N}$ status, lymphatic invasion, vascular invasion, perineural invasion, tumor budding, and tumor growth pattern (expanding or infiltrating type). Lymphatic invasion was identified by the presence of tumor cell clusters in endothelial lined spaces without a muscular coat. Vascular invasion was defined as tumor cell clusters in spaces with muscular coats. Perineural invasion was defined as cancerous cells spreading to the space surrounding a nerve. Tumor budding was defined as a cluster with a single cell or with 1 to 5 cells in the stroma around the tumor. Tumor budding was evaluated in 10 high power fields (HPFs) at high magnification $(\times 40)$ and was stratified as grade $1(0-4 / 10 \mathrm{HPF})$, grade 2 (5-9/10 HPF), grade 3 (10-19/10 HPF), and grade $4(\geq 20 / 10$ $\mathrm{HPF}$ ). Cases with tumor budding of grades $3-4$ ( $\geq 10 / 10 \mathrm{HPF})$ were considered positive [17]. Tumor growth patterns were classified into the expanding type, which grows by pushing the sur- 


\section{Annals of \\ Coloproctology Nari Shin, et al}

Cancer-Associated Fibroblasts and Desmoplastic Reactions Related to Cancer Invasiveness in Patients With Colorectal Cancer
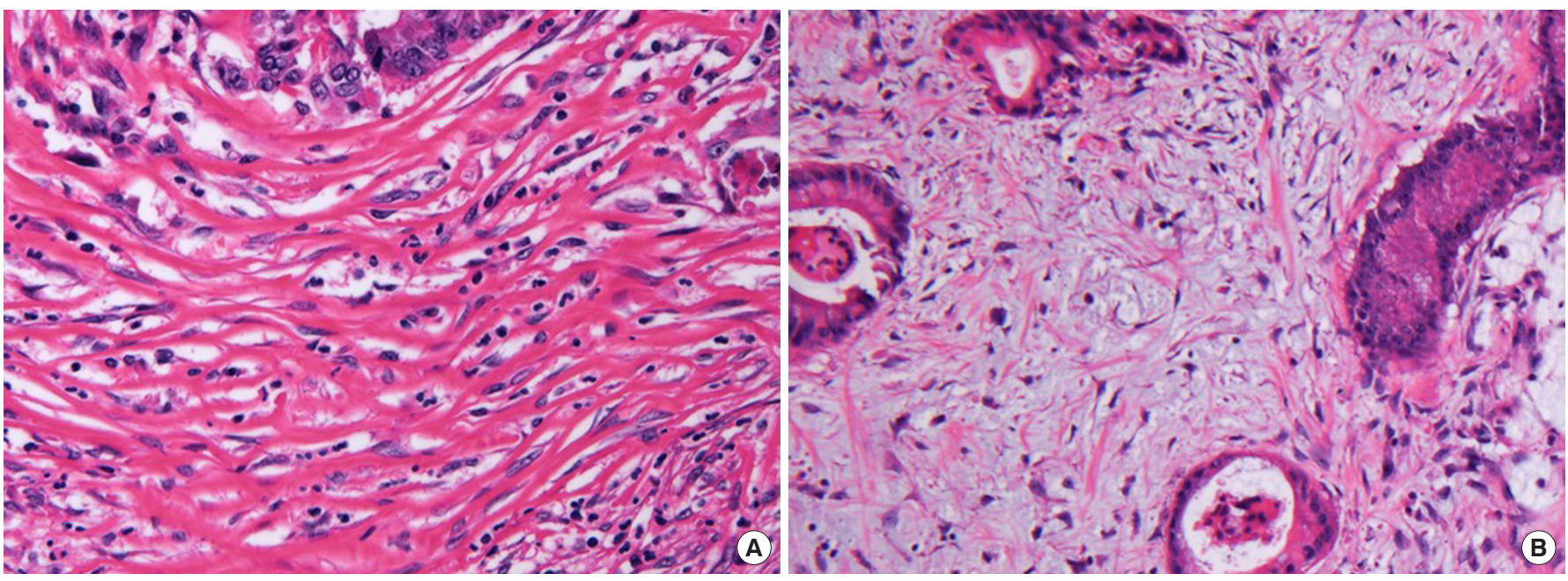

Fig. 1. Histological categorization of desmoplastic reactions and cancer-associated fibroblast (CAF) maturation. (A) Mature desmoplasia is composed of elongated collagen fibers and spindle-shaped CAF cells. (B) Immature desmoplasia is composed of myxoid components, keloidlike collagen bundles, and large plump CAFs $(\mathrm{H} \& \mathrm{E}, \times 40)$.

rounding stroma, and the infiltrating type, which grows by penetrating the stroma. Two pathologists reviewed the pathologic slides independently. When they had different views, they discussed and reevaluated the slides together. All pathological evaluations were performed using blinded clinical information.

\section{Patient follow-up}

We analyzed survival data and systemic recurrence of colorectal cancer patients during a follow-up of 3 years or longer. All patients underwent postoperative surveillance using abdominal and thoracic computed tomography and CEA measurements every 6 months. When cancer recurred at the site of the primary carcinoma, it was considered a local recurrence, and metastasis to other organs, such as the liver and lung, and intra-abdominal disseminated metastasis were considered systemic metastasis.

\section{Statistics and network analysis}

Chi-square and Pearson correlation tests were used to evaluate the correlation between the invasiveness characteristics of colon cancer and CAF maturation. The Kaplan-Meier method and the Log-Rank test were used to analyze the long-term oncologic outcomes. For multivariate analyses, the Cox proportional hazards model was used with forward stepwise selection. The covariance input criterion was $<0.1$, and the elimination criterion was $<0.05$. We also performed a network analysis between CAF maturation and desmoplastic reaction with cancer invasiveness. Network analyses were performed based on robust Spearman correlation (rho) measures for all collected parameters. The depth of each node indicates the number of edges. Positive $(r h o<0.2)$ and negative (rho $<-0.2)$ associations are represented as blue and red lines, respectively. Network visualization was conducted on the Gephi platform [18]. IBM SPSS Statistics ver. 24.0 (IBM Co., Armonk,
NY, USA) was used for statistics, and the significance level was $\mathrm{P}<$ 0.05 .

\section{RESULTS}

Desmoplastic responses appeared predominantly in the stroma in $78.3 \%$ of the colorectal cancer samples. Other histologic responses were inflammatory and mucinous reactions in $16.4 \%$ and $5.3 \%$ of the samples, respectively. The proportion of mature CAFs was $57.6 \%$ in the intratumoral stroma and $60.3 \%$ in the invasive front. No significant differences in clinicopathologic factors in cases with mature and immature CAFs were noted, except for EGFR expression (Table 1), which was significantly higher when mature CAFs were found in the invasive front (Fig. 2).

When patients were divided by stage, the incidence of lymphatic, vascular, and perineural invasion increased with increasing stage progression. Tumor budding was relatively frequent, even in the early stage. However, fibroblast maturation and tumor growth patterns were similar between the tumor stages (Table 2).

The correlation between fibroblast maturation and cancer invasiveness factors was analyzed. When mature fibroblasts were dominant in the intratumoral stroma, lymphatic invasion and expanding tumor growth were significantly more frequent. When immature fibroblasts prevailed in the invasive front, infiltrating tumor growth and advanced $\mathrm{T}$ status were significantly more common (Table 3). Tumor budding was observed in almost half of both groups, although it occurred more frequently in patients with tumors of an infiltrating type $(\mathrm{P}=0.003)$.

The 5-year overall survival rate was lower in the mature CAF group than in the immature CAF group, but this difference was not statistically significant. The survival difference by fibroblast maturation disappeared when the two groups were compared to 
Table 1. Patient characteristics $(n=151)$

\begin{tabular}{|c|c|c|c|c|c|c|}
\hline \multirow{2}{*}{ Characteristic } & \multicolumn{3}{|c|}{ Intratumoral CAF } & \multicolumn{3}{|c|}{ Invasive front CAF } \\
\hline & Mature $(\mathrm{n}=87)$ & Immature $(n=64)$ & P-value & Mature $(n=91)$ & Immature $(n=60)$ & P-value \\
\hline \multicolumn{7}{|l|}{ Clinical factors } \\
\hline Age, $\geq 70 \mathrm{yr}$ & $26(29.9)$ & 17 (26.6) & 0.655 & $26(28.6)$ & $17(28.3)$ & 0.975 \\
\hline Male sex & $46(52.9)$ & $42(65.6)$ & 0.116 & $51(56.0)$ & $37(61.7)$ & 0.493 \\
\hline $\mathrm{BMl}, \geq 25 \mathrm{~kg} / \mathrm{m}^{2}$ & $30(34.5)$ & $16(25.0)$ & 0.211 & $31(34.1)$ & $15(25.0)$ & 0.236 \\
\hline Serum CEA, $>5 \mathrm{mg} / \mathrm{dL}$ & $31(35.6)$ & $25(39.1)$ & 0.666 & $33(36.3)$ & $23(38.3)$ & 0.797 \\
\hline Colon obstruction & $30(34.5)$ & 27 (42.2) & 0.334 & $31(34.1)$ & $26(43.3)$ & 0.250 \\
\hline \multicolumn{7}{|l|}{ Cancer sideness } \\
\hline Right & $26(29.9)$ & $21(32.8)$ & 0.701 & $26(28.6)$ & $21(35.0)$ & 0.404 \\
\hline Left & $61(70.1)$ & $43(67.2)$ & & $65(71.4)$ & $39(65.0)$ & \\
\hline \multicolumn{7}{|l|}{ Pathologic factors } \\
\hline Tumor size, $>5 \mathrm{~cm}$ & $37(42.5)$ & $34(53.1)$ & 0.197 & $37(40.7)$ & $34(56.7)$ & 0.054 \\
\hline Pathologic stage & & & 0.154 & & & 0.095 \\
\hline I & $18(20.7)$ & $9(14.1)$ & & $20(22.0)$ & $7(11.7)$ & \\
\hline$\|$ & $26(29.9)$ & $24(37.5)$ & & $24(26.4)$ & $26(43.3)$ & \\
\hline III & $31(35.6)$ & 28 (43.3) & & 36 (39.6) & $23(38.3)$ & \\
\hline IV & $12(13.8)$ & $3(4.7)$ & & $11(12.1)$ & $4(6.7)$ & \\
\hline Histologic grade & & & 0.118 & & & 0.156 \\
\hline Well differentiated (G1) & $7(8.4)$ & $4(6.3)$ & & $9(10.5)$ & $2(3.3)$ & \\
\hline Moderately differentiated (G2) & $71(85.5)$ & $59(93.7)$ & & $73(84.9)$ & $57(95.0)$ & \\
\hline Poorly differentiated (G3) & $5(6.0)$ & $0(0)$ & & $4(4.7)$ & $1(1.7)$ & \\
\hline \multicolumn{7}{|l|}{ Molecular factors } \\
\hline Cytoplasmic CEA & $66(75.9)$ & $49(76.6)$ & 0.920 & $67(73.6)$ & $48(80.0)$ & 0.368 \\
\hline P53 accumulation & 55 (63.2) & $35(54.7)$ & 0.291 & $55(60.4)$ & $35(58.3)$ & 0.796 \\
\hline EGFR overexpression & $28(40.0)$ & $13(23.6)$ & 0.053 & $30(41.1)$ & $11(21.2)$ & 0.019 \\
\hline KRAS mutation & $21(32.3)$ & $18(32.7)$ & 0.961 & $21(30.4)$ & $18(35.3)$ & 0.574 \\
\hline BRAF mutation & $0(0)$ & $1(2.3)$ & 0.249 & $0(0)$ & $1(2.4)$ & 0.230 \\
\hline MSI-high & $8(15.7)$ & $5(11.4)$ & 0.541 & $10(18.5)$ & $3(7.3)$ & 0.116 \\
\hline
\end{tabular}

Values are presented as number (\%).

CAF, cancer-associated fibroblast; BMl, body mass index; CEA, carcinoembryonic antigen; EGFR, epidermal growth factor receptor; KRAS, K-ras proto-oncogene; BRAF, Braf proto-oncogene; MSI, microsatellite instability.

patients with nonmetastatic colorectal cancer. No differences in systemic recurrence were observed in the 2 fibroblast maturation groups (Table 4).

On univariate analyses using the Log-Rank test, T status, $\mathrm{N}$ status, lymphatic invasion, tumor budding, EGFR overexpression, old age, and elevated serum CEA were found to be prognostic factors for survival (Fig. 3). The factors associated with systemic recurrence were $\mathrm{T}$ status, $\mathrm{N}$ status, lymphatic invasion, vascular invasion, perineural invasion, old age, elevated serum CEA, and EGFR overexpression (Fig. 4). On multivariate analyses using the Cox proportional hazards model, lymph node metastasis and old age were independently associated with overall survival. T stage, old age, and EGFR overexpression were considered to be independent prognostic factors for systemic recurrence (Table 5).

Many prognostic factors other than EGFR are associated with CAF maturation. Therefore, we performed a network analysis to determine whether the various prognostic factors were correlated. In the network analysis using Spearman correlation coefficients, the critical nodes with more than five edges were lymphatic invasion, perineural invasion, $\mathrm{T}$ status, $\mathrm{N}$ status, colonic obstruction, and elevated CEA. Two well-connected islands were identified. One island included lymphatic invasion and vascular invasion, leading to aggressive phenotypes associated with advanced stages, large tumor size $(>5 \mathrm{~cm})$, obstruction, and lymph node metasta- 
sis. The second island was associated with EGFR overexpression, CAF maturation, and infiltrating tumor growth leading to tumor budding (Fig. 5).

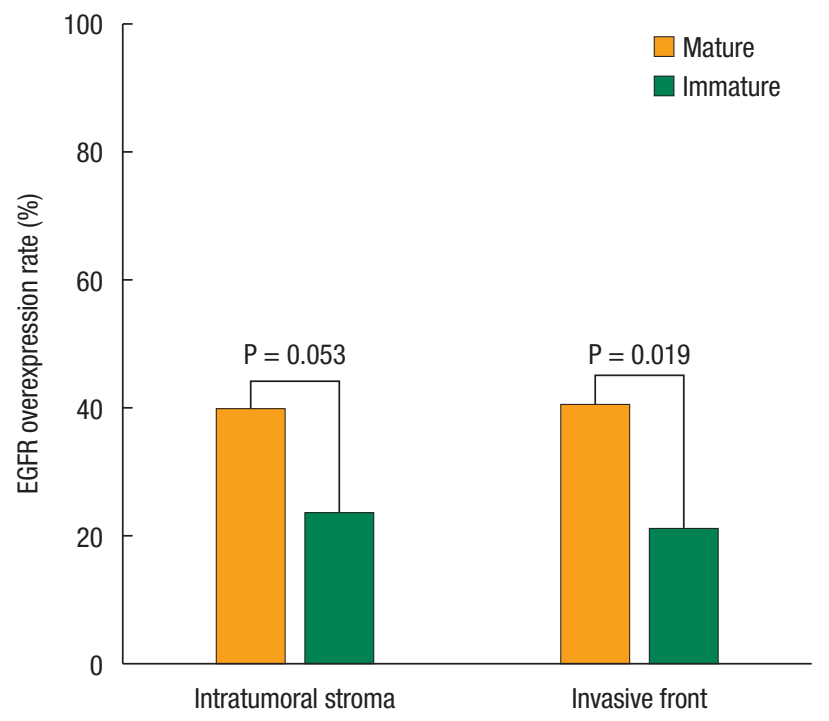

Fig. 2. Correlation of epidermal growth factor receptor (EGFR) overexpression and cancer-associated fibroblast maturation in the tumor stroma of colorectal cancer.

\section{DISCUSSION}

Dvorak [19] declared 30 years ago that cancer is a non-healing wound; since then, many studies of cancer invasion and metasta-

Table 2. Distribution of cancer-associated fibroblast (CAF) maturity and cancer invasiveness factors according to colorectal cancer stage $(\mathrm{n}=151)$

\begin{tabular}{lccccc}
\hline Variable & $\begin{array}{c}\text { Stage I } \\
(\mathrm{n}=27)\end{array}$ & $\begin{array}{c}\text { Stage II } \\
(\mathrm{n}=50)\end{array}$ & $\begin{array}{r}\text { Stage III } \\
(\mathrm{n}=59)\end{array}$ & $\begin{array}{r}\text { Stage IV } \\
(\mathrm{n}=15)\end{array}$ & P-value \\
\hline Intratumoral CAF & & & & & 0.154 \\
$\quad$ Mature & $18(66.7)$ & $26(52.0)$ & $31(52.5)$ & $12(80.0)$ & \\
$\quad$ Immature & $9(33.3)$ & $24(48.0)$ & $28(47.5)$ & $3(20.0)$ & \\
Invasive front CAF & & & & & 0.095 \\
$\quad$ Mature & $20(74.1)$ & $24(48.0)$ & $36(61.0)$ & $11(73.3)$ & \\
$\quad$ Immature & $7(25.9)$ & $26(52.0)$ & $23(39.0)$ & $4(26.7)$ & \\
Lymphatic invasion & $1(3.7)$ & $3(6.0)$ & $14(23.7)$ & $6(40.0)$ & 0.001 \\
Vascular invasion & $0(0)$ & $2(4.0)$ & $7(11.9)$ & $2(13.3)$ & 0.135 \\
Perineural invasion & $0(0)$ & $11(22.2)$ & $22(37.3)$ & $8(53.3)$ & $<0.001$ \\
Tumor budding & $8(29.6)$ & $23(46.0)$ & $35(59.3)$ & $8(53.3)$ & 0.076 \\
Tumor growth pattern & & & & & 0.389 \\
$\quad$ Expanding type & $9(33.3)$ & $11(22.0)$ & $10(16.9)$ & $4(26.7)$ & \\
$\quad$ Infiltrating type & $18(66.7)$ & $39(78.0)$ & $49(83.1)$ & $11(73.3)$ & \\
\hline
\end{tabular}

Values are presented as number (\%).

Table 3. Cancer-associated fibroblast (CAF) maturation and cancer invasion $(\mathrm{n}=151)$

\begin{tabular}{|c|c|c|c|c|c|c|}
\hline \multirow[b]{2}{*}{ Variable } & \multicolumn{3}{|c|}{ Intratumoral CAF maturation } & \multicolumn{3}{|c|}{ Invasive front CAF maturation } \\
\hline & $\begin{array}{l}\text { Mature CAF } \\
(\mathrm{n}=87)\end{array}$ & $\begin{array}{l}\text { Immature CAF } \\
\quad(n=64)\end{array}$ & P-value & $\begin{array}{l}\text { Mature CAF } \\
(\mathrm{n}=91)\end{array}$ & $\begin{array}{c}\text { Immature CAF } \\
(n=60)\end{array}$ & P-value \\
\hline Lymphatic invasion & $19(21.8)$ & $5(7.8)$ & 0.024 & $16(17.6)$ & $8(13.3)$ & 0.650 \\
\hline Vascular invasion & $8(9.2)$ & $3(4.7)$ & 0.358 & $6(6.6)$ & $5(8.3)$ & 0.754 \\
\hline Perineural invasion & $23(26.4)$ & $18(28.1)$ & 0.854 & $23(25.3)$ & $18(30.0)$ & 0.577 \\
\hline Tumor budding & $45(51.7)$ & $29(45.3)$ & 0.511 & $50(54.9)$ & $24(40.0)$ & 0.096 \\
\hline Infiltrating growth & $60(69.0)$ & $57(89.1)$ & 0.003 & $64(70.3)$ & $53(88.3)$ & 0.029 \\
\hline T status (3-4) & $61(70.1)$ & $49(76.6)$ & 0.460 & $60(65.9)$ & $50(83.3)$ & 0.024 \\
\hline N status (1-2) & $43(49.4)$ & $31(48.4)$ & 0.904 & $47(51.6)$ & $27(45.0)$ & 0.506 \\
\hline
\end{tabular}

Values are presented as number (\%).

Table 4. Five-year overall survival and systemic recurrence according to cancer-associated fibroblast (CAF) maturation in patients with colorectal cancer $(\mathrm{n}=151)$

\begin{tabular}{|c|c|c|c|c|c|c|c|c|}
\hline $\mathrm{CAF}$ & Overall survival & Odds ratio & $95 \% \mathrm{Cl}$ & P-value & Systemic recurrence & Odds ratio & $95 \% \mathrm{Cl}$ & P-value \\
\hline $\begin{array}{l}\text { Intratumoral CAF (mature vs. } \\
\text { immature) }\end{array}$ & $76.7 \%$ vs. $87.5 \%$ & 1.925 & $0.848-4.371$ & 0.118 & $23.9 \%$ vs. $29.1 \%$ & 0.921 & $0.486-1.746$ & 0.801 \\
\hline $\begin{array}{l}\text { Invasive front CAF (mature vs. } \\
\text { immature) }\end{array}$ & $77.9 \%$ vs. $88.3 \%$ & 2.060 & $0.876-4.847$ & 0.098 & $22.4 \%$ vs. $31.9 \%$ & 0.798 & $0.421-1.513$ & 0.489 \\
\hline
\end{tabular}

CAF, cancer-associated fibroblast; $\mathrm{Cl}$, confidence interval. 
sis have examined the wound healing process. During wound healing in cancer tissues, activated fibroblasts can lead to a normal wound healing response and serve as barriers to inhibit the spread of cancer cells; however, an atypical wound healing process similar to the keloid process can also occur in the tumor stroma, where excess corpulent fibers can be accumulated by plump fibroblasts. Immature fibrosis like a keloid scar can promote tumor infiltrating growth, and colon cancer patients with this phenotype may have an undesirable prognosis [5].

Tumor metastasis can be blocked by dense fibrous tissue. In current thought, the migration and invasion of cancer cells should be blocked by loose fibrotic tissue composed of myxoid and keloid- like collagen bundles and large plump CAFs. A cholangiocarcinoma with dense collagen walls around the cancer tissue indicates a good prognosis [20]. Additionally, colon cancer with high-density mature fibrosis suggests a favorable survival outcome [3]. Ueno et al. evaluated the fibrosis maturation of colorectal cancer patients and reported that the mature fibrosis group had a significantly higher survival rate, providing a pathological basis for the long-standing belief that the dense structure of mature fibrosis acts as an obstacle to tumor metastasis [3-6]. However, experimental studies have shown that CAF activation and fibrosis can promote cancer invasion. Activated fibroblasts produce abundant collagen fibers that form high-density fibrosis around cancer cells
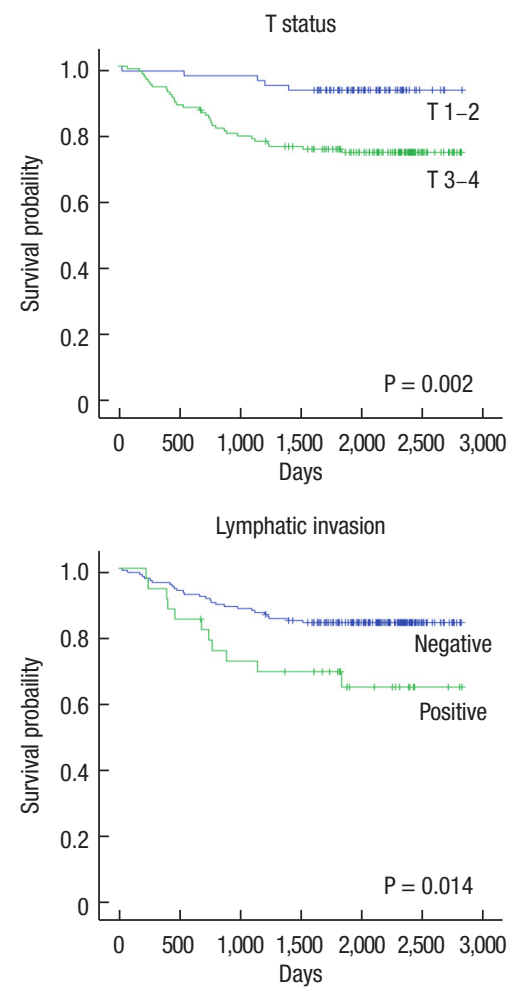

Age

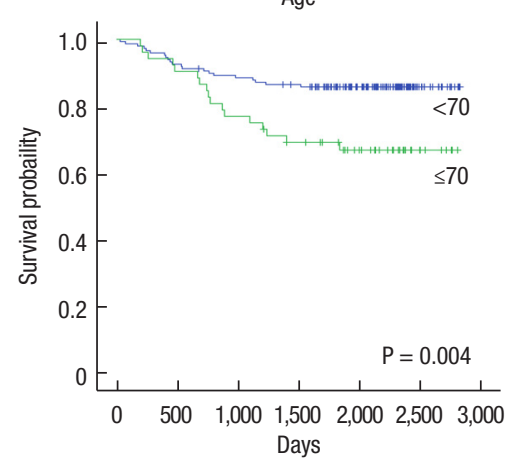

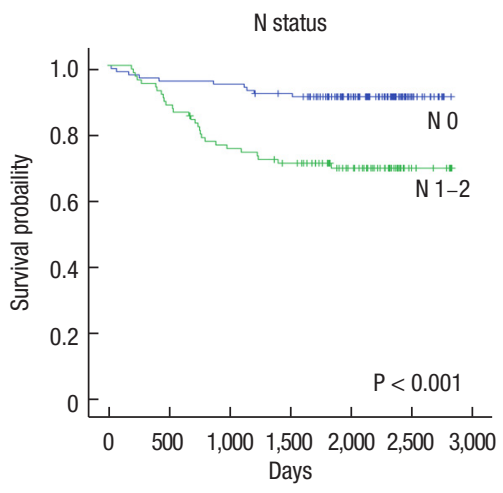

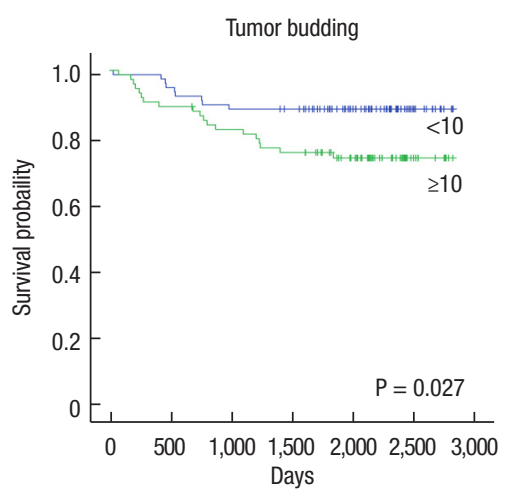

CEA

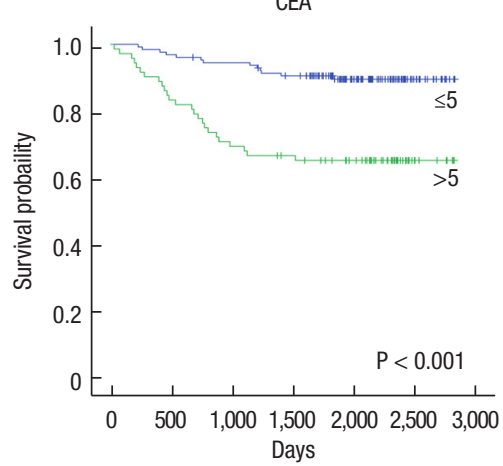

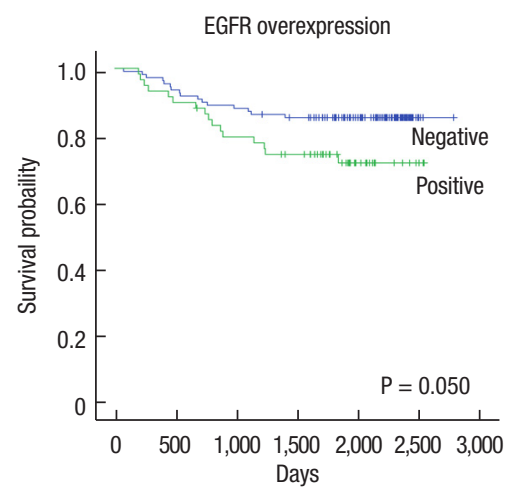

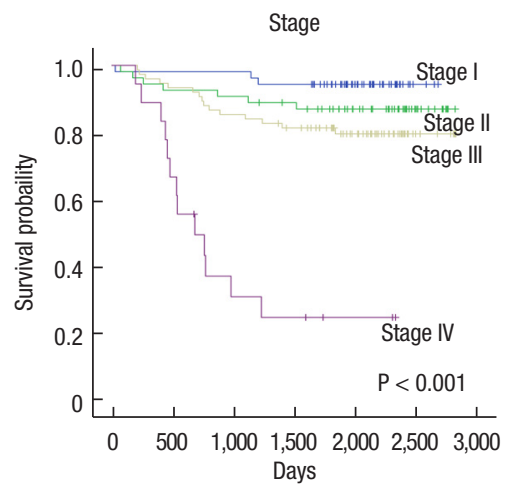

Fig. 3. Five-year overall survival of patients with colorectal cancer by significant prognostic factors. EGFR, epidermal growth factor receptor; CEA, carcinoembryonic antigen. 


\section{$\begin{array}{rlr}\text { Coloproctols of } & \text { With Colorectal } \\ \text { Cori Shin, et al. }\end{array}$}

\section{Cancer-Associated Fibroblasts and Desmoplastic Reactions Related to Cancer Invasiveness in Patients}
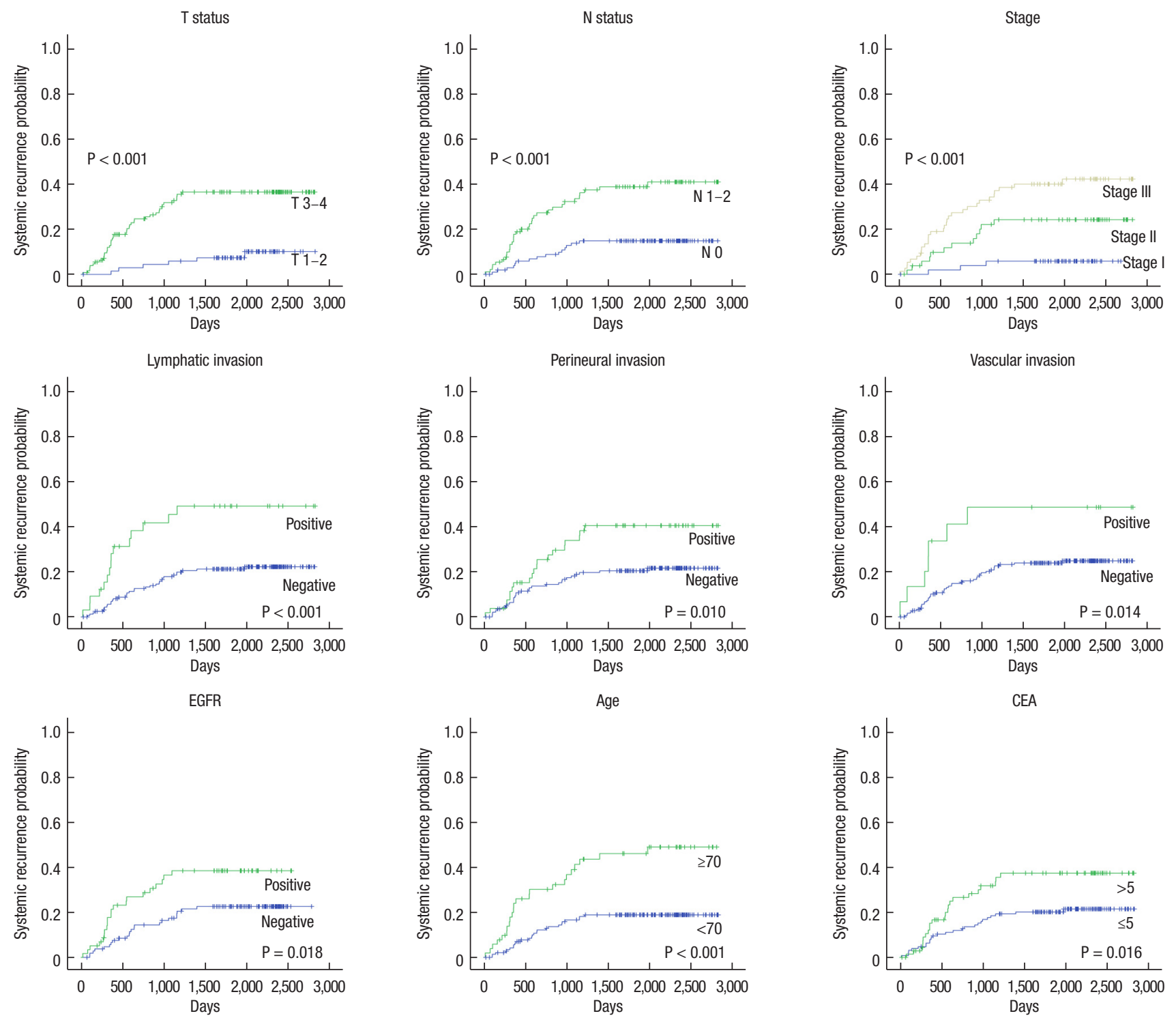

Fig. 4. Five-year systemic recurrence of colorectal cancer by significant prognostic factors. EGFR, epidermal growth factor receptor; CEA, carcinoembryonic antigen.

[21]. Fiber contraction and tumor stiffness in mature fibrotic tissue lead to cancer cell invasion in vitro [11-14]. Unlike the expectation that mature fibroblast responses will prevent the spread of cancer cells, these studies suggest that CAF activation can promote cancer invasion.

Recently, tumor stiffness has been experimentally proven to promote metastasis. When fibrosis forms a dense structure around cancer cell clusters in an adenocarcinoma, the internal pressure causes the gland structure to rupture, allowing the rapid spread of cancer cells [11]. In addition, the high-density fiber of the tumor stroma acts as a train track to promote the movement of cancer cells, thereby promoting invasion and metastasis [4].
Expression of lysyl oxidase (LOX), a factor involved in collagen arrangement during wound healing, can induce dense fibrosis. When LOX is activated in the tumor stroma and the collagen array is aligned, tumor stiffness promotes the migration, invasion, and metastasis of cancer cells [22]. The different results between clinicopathologic and experimental studies may be because of differences in the nature and the histological structure of various cancer cells.

Interestingly, in this study, the maturation of CAF has no relation to pathological stage. In the hypotheses when planning this study, the authors predicted that as cancer progressed, the proportion of immature CAF would increase and that these changes 
Table 5. Univariate and multivariate analyses of 5-year overall survival and systemic recurrence for patients with colorectal cancer $(\mathrm{n}=151)$

\begin{tabular}{|c|c|c|c|c|c|c|}
\hline \multirow{2}{*}{ Variable } & \multicolumn{3}{|c|}{ Univariate analysis } & \multicolumn{3}{|c|}{ Multivariate analysis } \\
\hline & $\mathrm{OR}$ & $95 \% \mathrm{Cl}$ & P-value & $\mathrm{OR}$ & $95 \% \mathrm{Cl}$ & P-value \\
\hline \multicolumn{7}{|l|}{ Overall survival } \\
\hline T status (3-4) & 4.014 & $1.566-10.235$ & 0.004 & - & - & - \\
\hline N status (1-2) & 3.654 & $1.774-7.530$ & $<0.001$ & 5.363 & $1.572-18.291$ & 0.007 \\
\hline Lymphatic invasion & 2.340 & $1.160-4.722$ & 0.018 & - & - & - \\
\hline Tumor budding & 2.386 & $1.079-5.275$ & 0.032 & 2.286 & $0.892-5.855$ & 0.085 \\
\hline EGFR overexpression & 0.506 & $0.253-1.013$ & 0.054 & - & - & - \\
\hline Age, $\geq 70 \mathrm{yr}$ & 2.455 & $1.295-4.656$ & 0.006 & 2.286 & $1.275-6.572$ & 0.011 \\
\hline Serum CEA, $>5 \mathrm{mg} / \mathrm{dL}$ & 4.075 & 2.083-7.971 & $<0.001$ & - & - & - \\
\hline \multicolumn{7}{|l|}{ Systemic recurrence } \\
\hline T status (3-4) & 5.037 & $2.141-11.850$ & $<0.001$ & 2.148 & $1.147-4.021$ & 0.017 \\
\hline N status (1-2) & 3.204 & $1.743-5.890$ & $<0.001$ & - & - & - \\
\hline Lymphatic invasion & 2.980 & $1.619-5.483$ & $<0.001$ & 2.815 & $1.487-5.330$ & 0.001 \\
\hline Perineural invasion & 2.087 & $1.179-3.693$ & 0.012 & - & - & - \\
\hline Vascular invasion & 2.634 & $1.182-5.871$ & 0.018 & - & - & - \\
\hline EGFR overexpression & 2.612 & 1.113-3.638 & 0.021 & 2.033 & $1.097-3.770$ & 0.024 \\
\hline Age, $\geq 70 \mathrm{yr}$ & 3.095 & $1.764-5.430$ & 0.001 & 2.148 & $1.147-4.021$ & 0.017 \\
\hline Serum CEA, $>5 \mathrm{mg} / \mathrm{dL}$ & 1.965 & $1.121-3.452$ & 0.018 & - & - & - \\
\hline
\end{tabular}

OR, odds ratio; $\mathrm{Cl}$, confidence interval; EGFR, epidermal growth factor receptor; CEA, carcinoembryonic antigen.

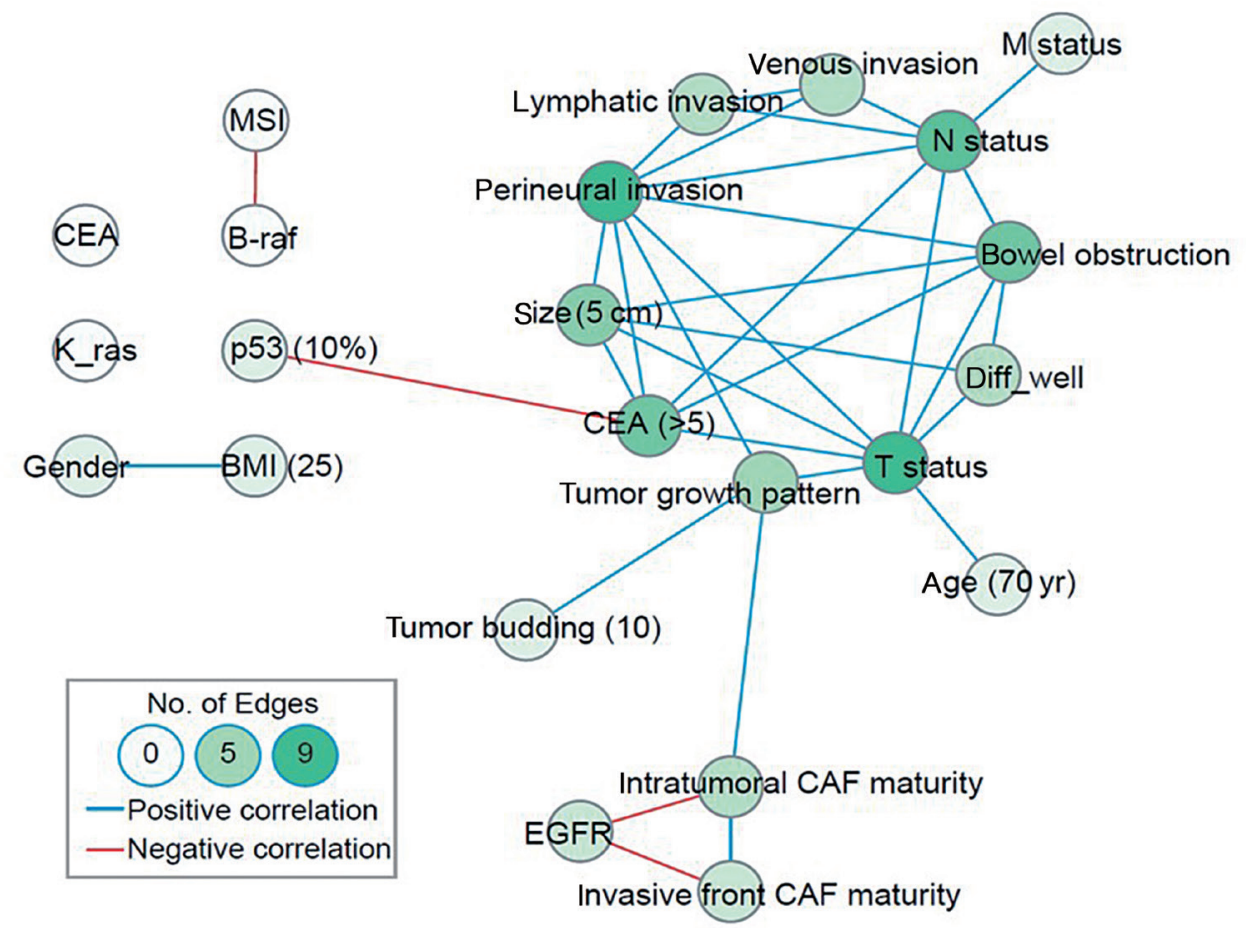

Fig. 5. Network analysis of Spearman's coefficients of correlation. The blue line reflects significant correlation with a coefficient $>0.2$. The red line reflects reverse correlation with a coefficient <-0.2. CEA, carcinoembryonic antigen; MSI, microsatellite instability; BMI, body mass index; EGFR, epidermal growth factor receptor; CAF, cancer-associated fibroblast. 
would be associated with poor prognosis. However, the results of the study showed that CAF maturation based on cytomorphologic characteristics was not statistically correlated with stage and showed no statistical difference as a prognostic factor for overall survival and systemic recurrence. A possibility is that the formation of the tumor microenvironment induced by cancer cells may be determined early in cancer development. Therefore, the immature CAF ratio would be presumed to be constant regardless of stage. Thus, to date, only few clinical studies have directly assessed the effects on cancer invasion of CAFs and desmoplastic responses in the pathologic tissues of colon cancer patients.

In this study, we analyzed the effects of fibrotic maturation of the tumor stroma on cancer invasiveness and long-term oncologic outcomes. Above all, no difference in 5-year survival was noted in patients with differing desmoplastic maturation states. However, we did see a difference in the mechanism of tumor invasion. When a mature desmoplastic reaction was observed, more lymphatic invasion had occurred. Conversely, immature fibrosis of the tumor stroma promoted infiltrating tumor growth. These various cancer invasion routes could be because of a mature or an immature stroma, which may dilute the prognostic effect of desmoplastic maturation.

Infiltrating tumor growth has been considered to be advantageous for cancer cell metastasis and is, therefore, a poor prognostic factor $[1,2]$. In this study, infiltrating tumor growth was observed prominently in cases of immature fibrosis in the invasive front. In contrast, a more expanding growth pattern was observed in cases with mature fibrotic reaction, consistent with the expectation that dense fibrosis acts as a physical barrier to cancer cell infiltration. However, the 5-year survival rate was similar in patients with infiltrating patterns and those with expanding growth patterns. These results suggest that different pathways of cancer invasion may be induced by different microenvironments, all leading to metastasis.

In the multivariate analyses, EGFR overexpression was found to be as an independent prognostic factor for systemic recurrence. Subanalyses showed that EGFR overexpression was also a statistically significant independent factor for systematic recurrence in the stage I-III nonmetastatic colorectal cancer patients (odd ratio, $2.445 ; 95 \%$ confidence interval, $1.278-4.674 ; \mathrm{P}=0.007$ ). Only 2 of 15 patients (13.3\%) with metastasis included in this study received EGFR inhibitor-based targeted therapy with the anti-EGFR drug Cetuximab (Erbitux, Merck, Darmstadt, Germany). Therefore, therapeutic effects, such as improvement of survival rate and inhibition of recurrence due to targeted treatment, including treatment with anti-EGFR drugs, were not likely to be important confounding factors in our analyses. When EGFR was strongly expressed in cancer tissue, CAF maturation in the invasive front was found in the dense desmoplasia and was associated with expanding tumor growth. In contrast, weak EGFR expression was consistent with immature CAFs and a loose fibrous stroma that was associated with infiltrating tumor growth. In an experimental study, the number of fibroblasts increased significantly in the presence of growth factors like epidermal growth factor (EGF) and basic fibroblast growth factor (bFGF), and EGF could stimulate fibroblasts to produce VEGF and hepatocyte growth factor (HGF) [23]. In another in vitro study, normal epithelial cells were transformed into activated fibroblasts through an epithelial-tomesenchymal transition by stimulation with transforming growth factor (TGF)- $\beta$ and FGF-2, promoting the invasion of cancer cells [24]. Therefore, fibroblast maturation can be controlled by using a variety of growth factors, such as EGF, FGF, and TGF, which are derived from cancer cells and can affect the growth pattern of cancer cells.

The invasion and the metastasis of cancer cells is a multi-step process that is caused by the complex interaction of various factors. In this study, 23 clinicopathologic and molecular factors were compared through network analyses. We were able to find 2 closely linked islands with different invasiveness characteristics. Thus, we could determine the correlation of various critical factors influencing the survival and the recurrence patterns of patients with colorectal cancer. The first pathway included the activation of various growth factors that could induce fibroblast maturation and increase tumor stiffness, leading to lymphatic invasion and vascular invasion, precipitating lymph node metastasis. In the second pathway, weak EGFR expression predominated in immature fibroblasts and a loose fibrous stroma could be formed, leading to infiltrating tumor growth and tumor budding.

This research has several limitations. Clinical data from a small number of patients in a single institution will have a statistically low power and require careful interpretation, so a larger scale study is needed. Moreover, the maturation and the activation of fibroblasts cannot be completely determined by using morphological evaluations. We used the criteria of Ueno et al. [3] for defining positive desmoplastic reactions and CAFs. Maturity evaluation using CAF shape and size has been applied in several previous pathologic research efforts. Typically, a fibroblast has a lanceolate form, but a myofibroblast, which is activated in the fibrosis process and is expressed by molecular markers such as smooth muscle actin (SMA), tends to become larger and plump. Based on the coincidence of cellular morphology and expression of molecular markers, Ueno et al. [3] were able to assess the activity of CAF easily through H\&E staining, and several important studies have demonstrated its value as an independent prognostic factor for patients with colorectal cancer. In addition, Dvorak [19] evaluated the maturity of desmoplastic reactions by applying pathological features such as abnormal wound healing, including keloid reactions and excessive fibrous deposition to the tumor microenvironment. However, cytomorphologic properties based on this H\&E staining have limited ability to assess CAF activation adequately. Thus, molecular studies are needed to reveal the mechanisms of fibroblast activation that affect cancer-cell invasion pathways.

Recently, fibrosis of submucosal cancers has been reported to be 
an important predictor of poor prognosis in patients with early colorectal cancer undergoing endoscopic mucosal resection [25]. Therefore, the expectation is that the prognosis for patients with early colorectal cancer can be predicted by using the characteristics of the tumor's microenvironment, and various studies are underway to develop an anti-CAF agent as a noble anticancer strategy. Therefore, molecular biologic parameters related to CAF activation are expected to be useful for clinical indications and for increasing the efficacy of new targeted therapies

In conclusion, the maturity of CAFs and desmoplastic reactions were associated with tumor infiltrating growth pattern and cancer invasion, such as lymphatic invasion and tumor budding. However, in this study, the cytomorphological characteristics of CAFs and desmoplastic reactions in H\&E stained tissues were insufficient for them to be used as independent prognostic factors for patients with colorectal cancer patients. Therefore, in the future, elaborate molecular biology research and large-scale clinical studies will be needed to apply the characteristics of CAFs to precision medicine and targeted therapy.

\section{CONFLICT OF INTEREST}

No potential conflict of interest relevant to this article was reported.

\section{ACKNOWLEDGMENTS}

This study was supported by a grant from the Research Institute for Convergence of Biomedical Science and Technology (612017-003), Pusan National University Yangsan Hospital.

\section{REFERENCES}

1. Conti J, Thomas G. The role of tumour stroma in colorectal cancer invasion and metastasis. Cancers (Basel) 2011;3:2160-8.

2. Shi Y, Du L, Lin L, Wang Y. Tumour-associated mesenchymal stem/stromal cells: emerging therapeutic targets. Nat Rev Drug Discov 2017;16:35-52.

3. Ueno H, Kanemitsu Y, Sekine S, Ishiguro M, Ito E, Hashiguchi Y, et al. Desmoplastic pattern at the tumor front defines poor-prognosis subtypes of colorectal cancer. Am J Surg Pathol 2017;41: 1506-12.

4. Ueno H, Shinto E, Shimazaki H, Kajiwara Y, Sueyama T, Yamamoto J, et al. Histologic categorization of desmoplastic reaction: its relevance to the colorectal cancer microenvironment and prognosis. Ann Surg Oncol 2015;22:1504-12.

5. Ueno H, Jones AM, Wilkinson KH, Jass JR, Talbot IC. Histological categorisation of fibrotic cancer stroma in advanced rectal cancer. Gut 2004;53:581-6.

6. Ueno H, Jones A, Jass JR, Talbot IC. Clinicopathological significance of the 'keloid-like' collagen and myxoid stroma in advanced rectal cancer. Histopathology 2002;40:327-34.
7. Kuzet SE, Gaggioli C. Fibroblast activation in cancer: when seed fertilizes soil. Cell Tissue Res 2016;365:607-19.

8. Guinney J, Dienstmann R, Wang X, de Reyniès A, Schlicker A, Soneson C, et al. The consensus molecular subtypes of colorectal cancer. Nat Med 2015;21:1350-6.

9. Ueno H, Mochizuki H, Hashiguchi Y, Shimazaki H, Aida S, Hase $\mathrm{K}$, et al. Risk factors for an adverse outcome in early invasive colorectal carcinoma. Gastroenterology 2004;127:385-94.

10. Mukaida N, Sasaki S. Fibroblasts, an inconspicuous but essential player in colon cancer development and progression. World J Gastroenterol 2016;22:5301-16.

11. Hanley CJ, Noble F, Ward M, Bullock M, Drifka C, Mellone M, et al. A subset of myofibroblastic cancer-associated fibroblasts regulate collagen fiber elongation, which is prognostic in multiple cancers. Oncotarget 2016;7:6159-74.

12. Malik R, Lelkes PI, Cukierman E. Biomechanical and biochemical remodeling of stromal extracellular matrix in cancer. Trends Biotechnol 2015;33:230-6.

13. Yu H, Mouw JK, Weaver VM. Forcing form and function: biomechanical regulation of tumor evolution. Trends Cell Biol 2011;21: 47-56.

14. Karagiannis GS, Poutahidis T, Erdman SE, Kirsch R, Riddell RH, Diamandis EP. Cancer-associated fibroblasts drive the progression of metastasis through both paracrine and mechanical pressure on cancer tissue. Mol Cancer Res 2012;10:1403-18.

15. Edge SB, Byrd DR, Compton CC, Fritz AG, Greene FL, Trotti A, editors. AJCC cancer staging manual. 7th ed. New York: Springer; 2010.

16. Pak MG, Lee CH, Lee WJ, Shin DH, Roh MS. Unique microRNAs in lung adenocarcinoma groups according to major TKI sensitive EGFR mutation status. Diagn Pathol 2015;10:99.

17. Koelzer VH, Zlobec I, Lugli A. Tumor budding in colorectal cancer--ready for diagnostic practice? Hum Pathol 2016;47:4-19.

18. Bastian M, Heymann S, Jacomy M. Gephi: an open source software for exploring and manipulating networks. Proc Third Int ICWSM Conf (ICWSM) 2009;8:361-2.

19. Dvorak HF. Tumors: wounds that do not heal. Similarities between tumor stroma generation and wound healing. N Engl J Med 1986;315:1650-9.

20. Zhang XF, Dong M, Pan YH, Chen JN, Huang XQ, Jin Y, et al. Expression pattern of cancer-associated fibroblast and its clinical relevance in intrahepatic cholangiocarcinoma. Hum Pathol 2017;65:92-100.

21. Wei SC, Yang J. Forcing through Tumor Metastasis: The Interplay between Tissue Rigidity and Epithelial-Mesenchymal Transition. Trends Cell Biol 2016;26:111-20.

22. Sakuma Y. Epithelial-to-mesenchymal transition and its role in EGFR-mutant lung adenocarcinoma and idiopathic pulmonary fibrosis. Pathol Int 2017;67:379-88.

23. Yu A, Matsuda Y, Takeda A, Uchinuma E, Kuroyanagi Y. Effect of EGF and bFGF on fibroblast proliferation and angiogenic cytokine production from cultured dermal substitutes. J Biomater Sci 


\section{Coloproctology Nari Shin, et al.}

Polym Ed 2012;23:1315-24.

24. Shirakihara T, Horiguchi K, Miyazawa K, Ehata S, Shibata T, Morita I, et al. TGF- $\beta$ regulates isoform switching of FGF receptors and epithelial-mesenchymal transition. EMBO J 2011;30: 783-95.
25. Youk EG, Sohn DK, Hong CW, Lee SD, Han KS, Kim BC, et al. Early outcomes of endoscopic submucosal dissection for colorectal neoplasms according to clinical indications. Dis Colon Rectum 2016;59:403-10. 